\title{
Long-span continuous rigid frame bridge construction control
}

\author{
Jianqiao Li \& Yaxun Yang \& Zhikui Yang \\ Collage of Highway,Chang'an University,Xi'an, China
}

\begin{abstract}
KEYWORD: Continuous rigid frame bridge;The cantilever construction;Linear control;Stress monitoring

ABSTRACT: Running is the cantilever method is adopted in the study of the construction of continuous rigid frame bridge construction monitoring. The bridge with high pier and large crossborder, narrow bridge deck and other characteristics, high construction requirement and great technical difficulties.According to these characteristics, an analysis of the mechanical characteristics of each construction stage.Establishing finite element model of each construction stage, compared with measured values of related parameters correction and error analysis. Thus established in this kind of bridge construction control, should be focusing on the deflection and stress monitoring, but also attaches great importance to the influence factors such as temperature, sunshine.
\end{abstract}

\section{INTRODUCTION}

Since the reform and opening up, China's transportation industry developing rapidly, and always focus on the development of industry in China.Especially after the subprime crisis of 2008, China introduced a series of measures.Roads, railways and become the main force of stimulating domestic demand, but also to China's transportation industry and traffic construction enterprise has brought the unprecedented development opportunity."Area" and "Asia investment banking", but also to the traffic construction enterprise has brought unprecedented opportunities and challenges.As the central and eastern regions more perfect of network, now China's transportation network is gradually extension and improvement to the western regions. To the western region, especially in southwest China needs a lot of Bridges to improve the traffic in this area.Mostly in southwest mountain hilly terrain, have high requirements for bridge structure and construction methods. So we need to carefully study the main points of the long-span bridge construction, construction monitoring accurately, to provide timely and effective quality related data.

\section{The research purpose and meaning}

For long-span bridge construction method is often more complex, the construction status is also more complex.For continuous rigid frame bridge is often the segmental cantilever construction method is adopted.In this way will inevitably lead to the change of the bridge structure internal force and displacement is more complicated.For prestressed concrete structures, the material itself is homogeneous, and will receive the influence of curing conditions such as temperature, humidity, age, and different segment will influence each other between the new and old concrete, and this effect and to present some uncertainty.So in the process of cantilever construction of continuous rigid frame bridge in construction, received the impact of these factors will inevitably cause each segment of the internal force and deformation deviates from the value of design to some extent.In order to guarantee the safety of the construction to ensure construction quality, at the same time, the status of the cost of the project in conformity with the design value as far as possible, must strict construction control on construction process. Ensure the bridge after stress and linear comply with the design requirements.

For continuous rigid frame bridge cantilever construction, the formation of it need to go through a complicated process.In the constructionIn the process of how to ensure the closed before two the lateral displacement deviation and the vertical deflection of cantilever end does not exceed allowable scope;How to ensure the closed after the bridge deck line good;How to avoid the construction of main girder section appears too much stress, itSome problems if not handled properly, not only would be adverse to the structure, and may make girder beam bottom curve is not smooth,Form a permanent defect which might affect the appearance beauty.In order to solve these problems, the only way is to the constructionProcess control. 
The bridge construction control that guarantee the safety of the bridge construction construction phase structure is obtained by the construction monitoringThe actual internal force and deformation, thus the complete tracking can master the construction process and development.If I found the constructionMeasured values and the theoretical calculation in the process of value differ too much, will check and analysis of the causes, and not inContinue to construction, otherwise it would be possible accidents.

\section{The main research contents in this paper}

For long-span continuous rigid frame bridge construction control mainly has the following several aspects: 1 deformation control: in construction process we will strictly control the vertical deflection and lateral displacement, ensure the completion of the construction of the next section can accurately.To ensure the implementation of the final closure with a bridge after the line is fluent and meet the design requirements; 2 : internal control in construction process in a timely manner in the key position using embedded monitoring the stress state of bridge strain gauge measures such as time, ensure the safety of construction, and can to a great extent, control the stress condition of state. This is especially important when the closure construction.

For prestressed concrete structures, in addition to the above reasons, concrete creep and shrinkage of the material such as relaxation properties of prestressed steel beam, and the change of ambient temperature, sunshine conditions cause since the temperature stress and temperature time internal forces caused by environmental factors such as construction conditions deviate from the design condition. We want to estimate the impact of these factors on the construction state, and be able to in the actual deviation value is greater than forecast is to analyze the reasons, put forward the countermeasures. To ensure the accuracy and security of the construction.

\section{The characteristics of the continuous rigid frame bridge cantilever construction}

\section{The characteristics of long span continuous rigid frame}

On mechanics of continuous rigid frame bridge with high flexible pier and girder consolidation in the form of a bridge structure, improve the integrity of the whole bridge and is advantageous to the seismic characteristics of the bridge, the top and bottom and joint force, make the internal force distribution is more advantageous to meet the requirements of the stress of the long-span bridge. And along the bridge to push stiffness is small, reduced the variety of additional load caused by the internal force influence; On the economy because of the long span continuous rigid frame internal force distribution is reasonable, so high beam compared with small span continuous beam, save material.And piers no bearing, don't need system conversion, saving construction cost; Continuous rigid frame bridge aesthetics on linear more fluent, particularly with mountainous environment coordination.

\section{The characteristics of the cantilever construction}

Cantilever construction is a kind of common phased construction method, in order for a typical frame bridge. 0 \# pier construction first, secondly erection block, cantilever construction in turn again, at this time for $\mathrm{T}$ structure form of statically determinate.Then complete the final closure construction, complete system transformation, and finally implemented as bridge state.Each phase of the work directly affect the next phase of construction, and irreversible effects on the state into a bridge.

\section{Continuous rigid frame bridge cantilever construction control method}

\section{Model is established in dispositif}

Should be taken before the construction of various construction mechanical state mechanics model is set up, by Midas Civil, Ansys finite element mechanics software to establish the corresponding mechanical model, determine the construction condition, calculate the ideally deformation and stress state of each construction stage.

Structural analysis of construction control methods including forward analysis, regression analysis and stress free state method.For segmental construction cantilever construction of 
prestressed concrete continuous rigid frame bridge, construction control structure calculation mainly adopts forward analysis and backward analysis.

a.Forward analysis:This calculation method is along with the advancement of construction stage, structure form, boundary constraint and load form is in constant change, the early stage of the structure of creep will occur, its geometric position also in change, so the structure of the previous phase state will be the basis of analyzing the construction phase structure, etc. We will the according to the order before and after the construction phase of structure analysis and calculation method is called the forward analysis. Specific to the advance of long span continuous rigid frame analysis.

Forward analysis can not only provide into the stress of the bridge structure with a more accurate result, also for the structural strength,Stiffness calculation, the determination of ideal state, but also for the construction phase to complete the bridge construction controlLay the foundation.

b.Regression analysis:In order to make after the completion of the structure design of the linear and the construction process by setting method in the process of the camber.For continuous rigid frame bridge construction by stages, general requirements given control points of each construction stage structuresHigh, in order to eventually make the structure satisfy the design requirements, this problem is difficult to solve with the method of forward analysis.Regression analysis can solve this problem, the basic idea is that assumption t0 structure internal force shut the time.Forward analysis results of t0 time, linear axis meet the design requirements. In the initial state, in accordance with the forward pointsThe inverse process analysis, to pour down the structure, analysis every time to dismantle a construction segment to the remaining structure influence, Within a phase analysis for the structure of the displacement and internal force state is the ideal state of construction phase structure.

\section{Parameter correction}

In establishing mechanical model, material parameters are adopted by the specification given by the design value, but in the actual construction due to different factors such as environment, construction details, cause the actual parameters and design values are different.At this time, should according to the actual situation or actual field test to measure the actual parameter values, the mechanics model.

a.In the structure design, structure design parameters are taken according to specification,Because the values of the design parameters of a part are generally less than the measured values, Therefore, in most cases the structure internal force and displacement is obtained by using the standard design parameters were measured in larger,It is designed to be safe, but cannot be ignored for structure construction control deviation, because it will directly affect the line and internal force of the construction conform to the design requirements.Therefore, dealing with part of the design parameters were determined in advance, in order to ensure the bridge into the bridge meet the design requirements after lay the foundation.b. The temperature parameter correction: Temperature is one of the main factors influencing the girder deflection. Temperature changes including seasonal temperature change and sunshine temperatureDegree of change, especially the sunshine temperature change will cause the temperature difference of main girder roof, floor, deflection in the main girder,At the same time, also can cause the temperature difference on both sides of the pier, the pier shafts have deviation.

\section{The error analysis}

Due to reasons such as in analysis of simplified model of active or unconscious to ignore some of the factors of reason, and indeed there are some uncertain factors, so the calculation model is always in a certain extent with the actual situation.In the event of a larger error, to analyze the cause of error.Timely correct mechanical model.If it is the construction problem, should immediately stop the construction.And take some countermeasures to ensure safety, and prepare for the next phase of construction.Design parameter error, construction error, measuring error, structural analysis model error caused by factors such as interference. 


\section{Level control principle}

In cantilever construction process of the main girder, the reasonable section of formwork elevation of beam, is related to the main girder of linear whether smooth, whether to meet the requirements of the design is an important problem. And the actual construction process, there are many factors will have difference with prior estimate of the ideal state (such as modulus of elasticity of concrete, concrete density, prestressed tension effect, etc.), and the deviation will inevitably impact on deformation of the bridge, the actual deformation and deformation theory has certain deviation, thus influence into a completed bridge elevation, deviate from the design intent.Elevation of the model is not equal to design the elevation, after completion of the bridge is always a certain camber, to offset the construction of all kinds of deflection, elevation calculation formula of the model are as follows:

$$
H_{l m i}=H_{s i j}+\sum f_{1 i}+\sum f_{2 i}+f_{3 i}+f_{4 i}+f_{5 i}+f_{g i}
$$

Type:

$$
\begin{aligned}
& H_{l m i} \text {--i segment elevation of the model; } \\
& H_{s i i} \text {--i segment design elevation; } \\
& \sum f_{1 i} \text {--Produced by the weight of each beam segment in the i section deflection of sum; } \\
& \sum f_{2 i} \text {--By various segmental prestressed tensioning in the i section deflection of sum; } \\
& f_{3 i} \text {--Concrete shrinkage and creep in the I section caused by deflection; } \\
& f_{4 i} \text {--The construction of the temporary load in the I section caused by deflection; } \\
& f_{5 i} \text {--Deflection caused by the use of the load in the I section; } \\
& f_{g i} \text {--Hanging basket deformation values. }
\end{aligned}
$$

Among them $\sum f_{1 i}, \sum f_{2 i}, f_{3 i}, f_{4 i}, f_{5 i}$ five of them in forward analysis and backward analysis and calculation,Hanging basket deformation value is based on a hanging basket load test, the test results, finally draw the hanging basket load --The deflection curve for interpolation.

\section{Continuous rigid frame bridge stress test and error analysis}

Due to the heterogeneity and instability of concrete materials, the selection of design parameters, construction status indeedAnd structure analysis model and so on many factors, the structure of the theoretical calculation of the stress and the measured stress is difficult to completelyAnastomosis. This is mainly because that the measured stress according to the actual strain of the concrete is calculated according to elastic theory relations.Come out, and the measured strain of concrete, both the stress and strain, also including shrinkage, creep, etc.Stress and strain, should will deduct the above the stress and strain, according to the stress of the elastic relationship obtained is more accurate, and about how to do this is a very complicated problem. So at the beginning of prestressed concrete continuousIn the structure of the bridge strain test, should through system identification, error analysis and processing, make as far as possible close to the measured stress. The theoretical calculation value, thus more accurate grasp of the structure of the real stress state.

\section{The conclusion}

In this paper, through theoretical analysis, the continuous rigid frame bridge cantilever construction linear control and stress monitoring are studied, summed up the following conclusions: 
a, Should pay attention to the construction of temporary load of the deflection of the beam body, its effect on the deflection of main girder during construction period, continuity and uncertainty and difficult to measure accurately.

b, Should attach great importance to the influence of temperature change on beam deflection.Measured data show that the effect of the temperature change and out of sync, with obvious lag effect.

\section{Summary}

Long-span continuous rigid frame bridge is a bridge is very common now.It is a kind of high order statically indeterminate structure, and exist in the construction process of system transformation.So the whole bridge in construction state and into a state of bridge has a completely different stress distribution. So we should attach importance to the construction condition of bridge alignment and stress control, achieve the forecast in advance. Once the test results do not tally with the model calculation results, we also can analyze the reason, to find countermeasures.For the prestressed concrete continuous rigid-frame bridge structure, we also consider the material, and the characteristics of the structure itself (such as material nonlinearity, time dependence), the internal forces and secondary internal forces of statically indeterminate structure, etc., comprehensive analysis, so as to ensure the accuracy of the construction.

\section{REFERENCES}

[1]Sheng Yang. The high piers large span continuous rigid frame bridge cantilever construction linear control and stress monitor research. [M]. Changsha, changsha university of science and technology, 2006.

[2]Fu Zhong. Bridge construction control technology [M]. Beijing,People's traffic press, 2001.

[3]Pingwei Zheng.Construction Control of Long-SPan Bridges [R].Wuhan, Bridge science research institute Ltd,China Zhongtie group,2009.

[4]Shizhong Qiu.Bridge construction control technique [M].Shanghai.Based engineering company in Shanghai special foundation design,2002.

[5]Hong Du.Continuous rigid frame bridge construction control.Chongqing.Chongqing traffic scientific research and design institute,2003.

[6]Xuesong Zhang.Long-span prestressed concrete continuous rigid frame bridge construction control theory and the practice.Chongqing jiaotong university,2001 